\title{
MODERN MEDICINE IN THE COVID-19 ERA
}

\author{
Jacek Smereka ${ }^{1,2}$ (D), Lukasz Szarpak ${ }^{2,3}$ (]) Krzysztof J. Filipiak ${ }^{4}$ \\ 'Wroclaw Medical University, Department of Emergency Medical Service, Wroclaw, Poland \\ ${ }^{2}$ Polish Society of Disaster Medicine, Warsaw, Poland \\ ${ }^{3}$ Comprehensive Cancer Center in Bialystok, Poland \\ ${ }^{4} 1^{\text {st }}$ Chair and Department of Cardiology, Medical University of Warsaw, Poland
}

Coronaviral infections have been known for a long time; these viruses cause a number of diseases, not only in humans but also in animals. The current SARS-CoV-2 pandemic started in 2019 in China $[1,2]$. Discussions are ongoing about the timing of the first case, but there is no doubt that it was during the last quarter of 2019. The virus infection can be non-specific and asymptomatic, mildly symptomatic, and can cause severe pneumonia and acute respiratory distress syndrome (ARDS) requiring mechanical ventilation, as well as septic shock and multiorgan failure.

Because modern medicine has not dealt so far with such a large number of infections that are dangerous for health care workers, conclusions should be drawn from the experience of other countries - at present, mainly China and Italy. The experience of physicians from these countries indicates the necessity of suitable organisation and management of patients with diagnosed or suspected SARSCoV-2 infection [1]. It is essential to take appropriate preventive measures and reduce the risk of infection. Sensible management of personal protective equipment and its correct use, as well as medical personnel training and proper work organisation, are crucial. We should remember that by following current recommendations we protect not only our patients but also ourselves and our families.

The preventive measures taken by medical personnel should be a priority because health care workers, especially physicians and nursing personnel experienced in caring for patients in a critical condition, constitute a key element in the fight against COVID-19 $[3,4]$. A ventilator can be manufactured relatively quickly, but educating a physician, a nurse, a paramedic, or a physiotherapist takes much longer. Wherever possible, infectious disease departments or hospitals (hospitals transformed into infectious single units) should be located in separate buildings or separate parts of buildings, with attention paid to communication routes.

Rapid identification and isolation of people suspected of SARS-CoV-2 infection is essential. Many authorities and organisations, including the world health organization (WHO), point out the crucial importance of performing as many tests as possible for early diagnosis of the infection in asymptomatic people or in those with only minor symptoms, in order to protect healthy people [3]. The 'stay at home' campaign is being undertaken on a large scale to reduce the risk of infection and thus the number of cases requiring to be addressed by the health care system. Regardless of how large a proportion of the population will recover from the infection and hopefully gain immunity against re-infection until an effective treatment and/or vaccine is developed, this will protect health care units from a dramatic increase in the number of COVID-19 patients requiring intensive treatment.

A typical image that may be encountered by a paramedic, physician, or nurse in the prehospital setting or emergency department is a patient who develops flu-like symptoms within 14 days of exposure. In particular, coughing, fever, headache, and muscular pains occur, and patients with a loss of smell and taste are also increasingly reported. It should be noted that some patients do not present dyspnoea at very low $\mathrm{SpO}_{2}$ values, often below $80 \%$; in the emergency department, the suspicion of COVID-19 is raised when carrying out imaging 
tests, even not directly on the lung tissue, for example in cases of orthopaedic injuries to the clavicle or shoulder. The characteristic radiological picture of the lungs or chest computed tomography (CT) indicates the suspicion of SARS-CoV-2 infection with very sparse clinical symptoms. The risk of myocarditis as a result of SARS-CoV-2 infection should also be noted.

Numerous individuals infected with SARS-CoV-2, up to $80 \%$, may have mild symptoms or be asymptomatic $[1,5]$. Medical personnel should pay special attention to pulsoxymetry - the measurement of arterial blood saturation, a reliable, fast, and non-invasive method. The role of pulsoxymetry cannot be overestimated, especially if the radiological picture of the lungs does not fully correlate with the patient's clinical condition and the physical examination reveals minor signs during lung auscultation. Oxygen therapy is essential to achieve satisfactory arterial blood saturation in the absence of significant hypercapnia. For emergency teams, it is important that passive oxygen therapy maintains $\mathrm{SpO}_{2}$ at a level of at least $90 \%$, and $92-95 \%$ in pregnant patients.

In the case of pneumonia, there are symptoms indicating damage to lung tissue. In some patients, the virus may induce an excessive immune response, which is manifested by increased cytokine concentrations and the so-called cytokine storm, leading to ARDS and severe respiratory failure.

It is particularly important to reduce the number of patients requiring mechanical ventilation. It should be emphasised that the problem does not only concern the number of ventilators, but also the capabilities of intensive care units. It is often forgotten that the ventilator itself does not solve the problem: a patient with severe respiratory failure requires intensive treatment, other equipment, and, above all, experienced medical staff. Therefore, the provision of an adequate number of intensive care unit (ICU) beds and medical personnel is crucial.

Some patients with severe respiratory failure require endotracheal intubation and mechanical ventilation. Quick tracheostomy is increasingly being suggested. The patient may also demand advanced renal replacement therapy, treatment with catecholamines, and targeted antibiotic therapy of coexisting infections. In cases of extremely severe respiratory failure, extracorporeal membrane oxygenation (ECMO) can be used, but because of the enormous workload involved in starting and continuing the treatment, with limited human resources and a lim- ited number of patients in whom ECMO can be applied, the method is rarely utilised in patients with COVID-19.

Work is ongoing on the possibility of using antivirals, malaria drugs, as well as steroids, immunoglobulins, and cytokine storm blockers.

Over the past few months, the pandemic has changed the lives of most people in Europe and around the world, causing hundreds of thousands of cases of the disease, and deaths counted in tens of thousands $[1,6,7]$. The pandemic is leading to enormous damage to the economy; millions of people are at risk of losing their jobs. This affects the political and economic situation as well as stability in the countries involved in the coronavirus pandemic. Many governments have taken protective measures to stabilise the economic situation and ensure stability, including, above all, health security $[8,9]$.

From the point of view of the health care system, particularly rescue and emergency medicine workers, the primary goal is treating and rescuing patients but also ensuring the safety of health care staff [5]. Information on procedures should be sought primarily from official announcements of the Ministry of Health; the medical press should be followed, and conclusions drawn from the observation of countries with a similar demographic and social structure and comparable economic and organisational health care system capacity in which the number of infections has been exceptionally high.

At this point, as we need to listen to our Chinese colleagues, who managed the first wave of the pandemic. It might be important to cite George Gao, director-general of the Chinese Centre for Disease Control and Prevention, interviewed by Sciencemag. org, on March $27^{\text {th }}, 2020$. He said: "The big mistake in the U.S. and Europe, in my opinion, is that people aren't wearing masks. This virus is transmitted by droplets and close contact. Droplets play a very important role - you've got to wear a mask, because when you speak, there are always droplets coming out of your mouth. Many people have asymptomatic or presymptomatic infections. If they are wearing face masks, it can prevent droplets that carry the virus from escaping and infecting others" [10]. Paramedics and all the other medical staff, at this stage of the pandemic, should spread this message.

Acknowledgements: Article was supported by the Polish Society of Disaster Medicine. 
Conflict of interest: The authors declare that they have no conflict of interest.

\section{REFERENCES}

1. Baden LR, Rubin EJ. Covid-19 - The Search for Effective Therapy. N Engl J Med. 2020 [Epub ahead of print], doi: 10.1056/NEJMe2005477, indexed in Pubmed: 32187463.

2. Grasselli G, Pesenti A, Cecconi M. Critical Care Utilization for the COVID-19 Outbreak in Lombardy, Italy: Early Experience and Forecast During an Emergency Response. JAMA. 2020 [Epub ahead of print], doi: 10.1001/jama.2020.4031, indexed in Pubmed: 32167538.

3. Jones DS. History in a Crisis - Lessons for Covid-19. N Engl J Med. 2020 [Epub ahead of print], doi: 10.1056/NEJMp2004361, indexed in Pubmed: 32163699.

4. Livingston E, Bucher K. Coronavirus Disease 2019 (COVID-19) in Italy. JAMA. 2020 [Epub ahead of print], doi: 10.1001/jama.2020.4344, indexed in Pubmed: 32181795.

5. Murthy $S$, Gomersall $C D$, Fowler RA. Care for Critically III Patients With COVID-19. JAMA. 2020 [Epub ahead of print], doi: 10.1001/ jama.2020.3633, indexed in Pubmed: 32159735.
6. Smereka J, Szarpak L. COVID 19 a challenge for emergency medicine and every health care professional. The American Journal of Emergency Medicine. 2020, doi: 10.1016/j.ajem.2020.03.038.

7. Stebbing J, Phelan A, Griffin I, et al. COVID-19: combining antiviral and anti-inflammatory treatments. The Lancet Infectious Diseases. 2020; 20(4): 400-402, doi: 10.1016/s1473-3099(20)30132-8.

8. Wu Z, McGoogan JM. Characteristics of and Important Lessons From the Coronavirus Disease 2019 (COVID-19) Outbreak in China: Summary of a Report of 72314 Cases From the Chinese Center for Disease Control and Prevention. JAMA. 2020 [Epub ahead of print], doi: 10.1001/jama.2020.2648, indexed in Pubmed: 32091533.

9. Zu ZiY, Jiang MDi, Xu PP, et al. Coronavirus Disease 2019 (COVID-19): A Perspective from China. Radiology. 2020 [Epub ahead of print]: 200490, doi: 10.1148/radiol.2020200490, indexed in Pubmed: 32083985.

10. https://www.sciencemag.org/news/2020/03/not-wearing-masksprotect-against-coronavirus-big-mistake-top-chinese-scientist-says (27.03.2020). 